\title{
»War on drugs« und Verfassung - Plädoyer für die Abschaffung des Brechmitteleinsatzes im Strafverfahren
}

Bereits seit einigen Jahren wird die verfassungsrechtliche Zulässigkeit des Einsatzes von Brechmitteln als strafverfahrensrechtliche Ermittlungsmaßnahme kontrovers diskutiert. Meist werden Brechmittel gegenüber Personen eingesetzt, die des Straßendrogenhandels verdächtig sind. Ein Todesfall in Bremen zwingt dazu den Brechmitteleinsatz auf den verfassungsrechtlichen Prüfstand zu stellen.

\section{Kein »tragischer Einzelfall«}

Am 6.1.2005 starb ein 35-jähriger Mann an den Folgen eines gewaltsamen Brechmitteleinsatzes. Dem von Polizeibeamten gefesselten und sich wehrenden Verdächtigen waren von einem Polizeiarzt gewaltsam Brechmittel und große Mengen Wassers durch einen Schlauch in den Magen gepumpt worden, um dort vermutete verschluckte Kokain-Kugeln sicherzustellen. Die rechtsmedizinische Untersuchung des Toten kam zu dem Ergebnis, dass dieser durch den Brechmitteleinsatz quasi ertränkt wurde, nachdem große Wassermengen in die Lunge eingedrungen waren. ${ }^{1}$

Der Bremer Innensenator Röwekamp nannte den Todesfall einen »tragischen Einzelfall «. ${ }^{2}$ Er will auch nach diesem Vorfall an dem Brechmitteleinsatz grundsätzlich festhalten. »Schwerstkriminelle« müssten »mit körperlichen Nachteilen« rechnen. Seit 1992 sei allein in Bremen fast 1000 Mal Brechmittel ohne Beanstandung eingesetzt worden. ${ }^{3}$ Er halte es für völlig gerechtfertigt, mit unnachgiebiger Härte gegen solche Leute, die Drogen gewerbsmäßig verkauften, vorzugehen und dann müssten sie eben auch in Kauf nehmen, dass ihnen ein Brechmittel verabreicht werde. Den Schaden habe sich der Betroffene selbst zuzuschreiben. ${ }^{4}$

Auch die Gewerkschaft der Polizei fordert unbedingt an der bisherigen Praxis festzuhalten. $^{5}$

Verschwiegen wird, dass bereits im Jahr 2001 ein 19-jähriger Tatverdächtiger in Hamburg durch einen gewaltsamen Brechmitteleinsatz ums Leben gekommen ist. Einige Bundesländer verzichten seitdem auf den Einsatz von Brechmitteln. Auch Bremen hat den Einsatz von Brechmitteln inzwischen nach dem jüngsten Todesfall auf Grund des immer stärker werdenden öffentlichen Drucks auf Innensenator Röwekamp - zumindest vorübergehend - gestoppt ${ }^{6}$. Andernorts geht der Brechmitteleinsatz unter Billigung großer Teile der Rechtsprechung ungehindert weiter. ${ }^{7}$

1 Frankfurter Rundschau v. 8. u. 10.1.2005; Die Tageszeitung v. 6. u 7.1.2005.

2 Frankfurter Rundschau v. 8.1.2005.

3 Frankfurter Rundschau v. 10.1.2005.

4 Die Tageszeitung v. 4., 6. u. 7.1.2005.

5 Die Tageszeitung v. 8.1.2005.

6 Frankfurter Rundschau v. 25.1.2005.

7 Frankfurter Rundschau v. 6.1.2005. 


\section{Verfassungsrechtliche Bedenken}

Beim Brechmitteleinsatz handelt es sich um eine der umstrittensten strafprozessualen Ermittlungsmethoden. Die bisher überwiegende Auffassung in der Rechtsprechung und im strafrechtswissenschaftlichen Schrifttum hält den Brechmitteleinsatz unter Einhaltung der gesetzlichen Vorgaben und unter Wahrung des Verhältnismäßigkeitsprinzips für verfassungsgemäß. ${ }^{8}$

Er ist als Ermittlungsmaßnahme in der StPO ausdrücklich nicht geregelt. Die überwiegende Auffassung zieht als Ermächtigungsgrundlage $§ 81$ a Abs.1 StPO heran. ${ }^{9}$

Entgegen anderes lautender Behauptungen muss hervorgehoben werden, dass das BVerfG bisher nicht über die Frage der Verfassungsmäßigkeit des Brechmitteleinsatzes entschieden hat. Von einigen Befürwortern des Brechmitteleinsatzes wird eine Entscheidung des Gerichts aus dem Jahre 1999 dahingehend interpretiert, dass das Gericht den Brechmitteleinsatz für verfassungsgemäß erklärt habe. ${ }^{10}$ Das BVerfG hatte im Rahmen eines Nichtannahmebeschlusses in einem Nebensatz ausgeführt, dass der Brechmitteleinsatz »im Hinblick auf die durch Art. 1 Abs.1 GG geschützte Menschenwürde und den in Art. 2 Abs.1 i.V.m. Art.1 Abs.1 GG enthaltenen Grundsatz der Selbstbelastungsfreiheit grundsätzlichen verfassungsrechtlichen Bedenken nicht begegnet. $\ll^{11}$ Diese Ausführungen legen auf den ersten Blick den Eindruck nahe, dass das Gericht den Brechmitteleinsatz für verfassungsgemäß erklärt habe; tatsächlich durfte das BVerfG jedoch im Rahmen eines Nichtannahmebeschlusses keine inhaltlichen Feststellungen zur Verfassungsmäßigkeit des Brechmitteleinsatzes vornehmen. ${ }^{12}$ Es ist im übrigen nicht vorstellbar, dass das BVerfG ohne mündliche Anhörung des Betroffenen in einem bloßen Nebensatz einer Nichtannahmeentscheidung zur Verfassungsmäßigkeit einer solch umstrittenen Ermittlungsmaßnahme Stellung nimmt, weshalb der Satz im wissenschaftlichen Schrifttum zu Recht für rechtlich bedeutungslos gehalten wird. ${ }^{13}$ In einem bemerkenswerten Vorgang sah sich das BVerfG nach dem Hamburger Todesfall genötigt, mittels einer Pressemitteilung klarzustellen, dass es zur Verfassungsmäßigkeit des Brechmitteleinsatzes bisher nicht Stellung genommen habe. Es sei insbesondere noch nicht entschieden, ob eine zwangsweise Verabreichung mit Blick auf den Schutz der

8 KG, JR 2001, 162 m. krit. Anm. Hacketal; OLG Bremen, NStZ-RR 2000, 270; Benfer, JR 1998, 53; Beulke, Strafverfahrensrecht (2001) Rn.241; Birkholz u.a., Kriminalistik 1997, 277; LR-Dahs (1997), § 81a Rn.16; Fahl, JuS 2001, 47 (52); Götz M., JuS 2000, 727; Grüner, JuS 1999,122; Körner, StV 1988, 448; Meyer-Goßner, StPO (2003), § 81a Rn.22; Pfeiffer, StPO (2005), § 81a Rn.1; Rogall, NStZ 1998, 66; Schaefer, NJW 1997, 2437; Schroeder, Strafprozessrecht (2001) Rn.109; Weidemann, JA 2003, 328 (330).

9 vgl. Fn.7.

10 Vgl. etwa: Bachmann/ Püschel/ Sonnen, Kriminalistik 2004, 678 (680); Meyer-Goßner, StPO (2003),§ 81a Rn.22; SK-StPO-Rogall (2000), § 81a Rn.48.

11 BVerfG, StV 2000, 1.

12 So auch: Rixen, NStZ 2000, 381 (382) »Die Kammern des Verfassungsgerichts sind nicht befugt, unabhängig vom Verfahrensgegenstand und Verfahrensnotwendigkeit Rechtsmeinungen zu verbreiten.«

13 Naucke, StV 2000, 1 ff.; Rixen, StV 2000, 381 f. und ihnen folgend: Binder/ Seemann, NStZ 2002, 234 (234); Dallmeyer, KritV 2000, 252 f.; Hacketal, JR 2001, 164; Kühne, Strafprozessrecht (2003) S.247; Zaczyk, NStZ 2002, 125. 
körperlichen Unversehrtheit (Art.2 Abs.2 GG) und auf die Verhältnismäßigkeit des Eingriffs zulässig ist. ${ }^{14}$ Diese Ausführungen werden mit Recht dahingehend interpretiert, dass das Gericht die Verfassungsmäßigkeit des Brechmitteleinsatzes für fraglich hält und eine erneute Verfassungsbeschwerde anregen möchte. ${ }^{15}$

Und in der Tat werden gegen den gewaltsamen Brechmitteleinsatz gewichtige verfassungsrechtliche Einwände erhoben, welche einen künftigen Verzicht auf diese umstrittene Maßnahme zwingend gebieten.

\section{Verstoß gegen das Recht auf körperliche Unversehrtheit}

Die Brechmittelvergabe hat das Ziel, dass sich die Betroffenen massiv übergeben müssen, damit auf diese Weise im Magen vermutete Rauschgift-Kügelchen als Beweismittel erlangt werden können. In der Regel werden zu diesem Zweck die Brechmittel Ipecacuanha oder Apomorphin eingesetzt. ${ }^{16}$ Bei Ipecacuanha handelt es sich um einen flüssigen Sirup der vom Verdächtigen entweder freiwillig getrunken werden muss oder anderenfalls unter Gewaltanwendung mittels einer Magensonde durch die Nase verabreicht wird. Apomorphin wird mittels einer Injektion dem Körper zugeführt. ${ }^{17}$

Die zwangsweise Brechmittelvergabe stellt einen rechtfertigungsbedürftigen Eingriff in das Grundrecht auf körperliche Unversehrtheit des Art.2 Abs.2 S.1 GG dar. Die Rechtfertigungsanforderungen werden dem Verhältnismäßigkeitsprinzip entsprechend um so strenger, je intensiver der Grundrechtseingriff ist. Geringfügige Eingriffe in die körperliche Unversehrtheit zum Zwecke der Strafverfolgung wie etwa die Abnahme einer Blutprobe sind regelmäßig zulässig.

Als Ermächtigungsgrundlage wird - wie bereits angeführt wurde - in der Regel § 81a Abs.1 StPO angeführt. Nach dieser Vorschrift darf eine körperliche Untersuchung des Beschuldigten zur Feststellung von Tatsachen angeordnet werden, die für das Verfahren von Bedeutung sind. Zu diesem Zwecke sind neben der Blutentnahme auch andere körperliche Eingriffe zulässig, die von einem Arzt nach den Regeln der ärztlichen Kunst vorgenommen werden, wenn kein Nachteil für die Gesundheit des Verdächtigen zu befürchten ist.

$\S 81$ a StPO wird überwiegend für verfassungsgemäß gehalten. ${ }^{18}$ Diese Vorschrift ist Ausdruck einer normativen Abwägung zwischen dem öffentlichen Strafverfolgungsinte-

14 Vgl. Pressemitteilung des BVerfG Nr.116/ 2001 v. 13.12.2001.

15 Zaczyk, NStZ 2002, 125.

16 Vgl. OLG Frankfurt, NJW 1997, 1647.

17 Vgl. hierzu im Überblick: Dettmeyer u.a., MedR 2000, 316 (319). Laut Wesslau, StV 1997, 341 (342) ist der Einsatz von Ampomorphin inzwischen zumindest in Bremen und Hessen per Rundverfügung auf Grund medizinischer Bedenken untersagt worden.

18 Vgl. BVerfGE 16, 194 (201); 17, 108 (117); 47, 239 ff.; Pfeiffer, StPO (2005), § 81a Rn.1; Badura, Staatsrecht (2003) S.128; vgl. aber im Hinblick auf die weitgehende Unbestimmtheit der Vorschrift und unter dem Blickwinkel einer Beeinträchtigung der Unschuldsvermutung: Sax in: Bettermann/ Nipperdey/ Scheuner, Die Grundrechte (1959), Bd.III/2 S.986.; kritisch auch: Geerds, GA 1965, 321 ff., Rossmanith, Die Verfassungsmäßigkeit von körperlichen Eingriffen nach § 81a StPO (1969); vgl. ferner die Nachweise bei: Neumann, FS für E.A. Wolff (1998) S.373 (375) u. S.384, der ferner mit Recht hervorhebt, dass in zahlreichen anderen Staaten zwangsweise durchgeführte Eingriffe in die körperliche Unversehrtheit für unzulässig gehalten werden. 
resse und den Grundrechten der Bürger. ${ }^{19}$ Da die Vorschrift Eingriffe in die körperliche Unversehrtheit bereits bei einfachem Tatverdacht zulässt, ${ }^{20}$ sind strenge Anforderungen an die Verhältnismäßigkeit zu fordern, wie auch das BVerfG hervorhebt. ${ }^{21}$

Im Zeitpunkt des Ermittlungsverfahrens gilt die Unschuldsvermutung. Die Bürger trifft aber eine »prozessuale Aufopferungspflicht ${ }^{22}{ }^{2}$ Sie müssen die weitere Sachverhaltsaufklärung seitens der Strafverfolgungsorgane dulden. Es gibt jedoch Grenzen: Die Bürger sind im Hinblick auf ihr Grundrecht auf körperliche Unversehrtheit zur Duldung von strafprozessualen Ermittlungsmaßnahmen nur soweit verpflichtet, wie mit diesen zur Sachverhaltsaufklärung erforderlichen Eingriffen keine Nachteile für die Gesundheit verbunden sind.

Solche Nachteile müssen mit an Sicherheit grenzender Wahrscheinlichkeit ausgeschlossen werden können. Bereits eine gewisse Möglichkeit des Eintritts von körperlichen Nachteilen schließt die Zulässigkeit des Eingriffs aus. ${ }^{23}$ Beeinträchtigungen, die sich auf die Zeit des Eingriffs beschränken, sollen allerdings nicht erfasst sein. Ein körperlicher Nachteil im Sinne von $\S$ 81a Abs.1 StPO liegt somit bei nicht unerheblichen über die Dauer des Eingriffs hinausgehenden Beeinträchtigungen des körperlichen Wohlbefindens vor. ${ }^{24}$

Nun könnte man bereits auf Grund der eingetretenen Todesfälle feststellen, dass der Brechmitteleinsatz unzulässig sein müsste. Etwas anderes könnte dann gelten, wenn sich in den Todesfällen, wie gelegentlich behauptet wird, nicht ein dem Brechmitteleinsatz innewohnendes Gesundheitsrisiko verwirklicht hat. Es ist somit im Hinblick auf die Vereinbarkeit des Brechmitteleinsatzes mit dem Grundrecht auf körperliche Unversehrtheit aus Art.2 Abs.2 S.2 GG im folgenden zu klären, mit welchen Beeinträchtigungen des körperlichen Wohlbefindens und mit welchen gesundheitlichen Risken ein mit Zwang durchgeführter Einsatz verbunden ist.

Durch die Verabreichung von Brechmittel wird beim Betroffenen ein ganz massiver Brechreiz hervorgerufen. Der Betroffene muss sich - auch wenn der Einsatz des Mittels ohne Komplikationen verläuft - meist mehrfach übergeben. Berichten zu folge, klagen viele Betroffene über langandauernde Müdigkeit, Übelkeit und Durchfall sowie tagelanges Erbrechen. ${ }^{25}$ Auch die Befürworter eines solchen Vorgehens räumen ein, dass diese Ermittlungsmaßnahme mit erheblichen Beeinträchtigungen des

19 Ziel ist die Herstellung praktischer Konkordanz, vgl. Hesse, Grundzüge des Verfassungsrechts der Bundesrepublik Deutschland (1995) S.142.

20 Kritisch: Sax ebd.

21 BVerfGE 17, 108 (117).

22 Grundlegend: Frister, Schuldprinzip, Verbot der Verdachtsstrafe und Unschuldsvermutung als materielle Grundprinzipien des Strafrechts (1988) S.121 ff.; Köhler, ZStW 107 (1995) S.10, 21 ff., Neumann, FS für E.A.Wolff (1998) S.391.

23 Eisenberg, Beweisrecht der StPO (2002) Rn.1635; Meyer-Goßner, StPO (2003), § 81a Rn.17.

24 Eisenberg, Beweisrecht der StPO (2002) Rn.1635; Grüner, JuS 1999, 122 (123); MeyerGoßner, StPO (2003) § 81a Rn.17; Schlüchter, Das Strafverfahren (1983) S.176.

25 Vgl. Birkholz u.a., Kriminalistik 1997, 277 (279) langandauerndes Erbrechen in 17,1 \% der untersuchten Fälle; Wesslau, StV 1997, 341 (344); Roggan, Bürgerrecht \& Polizei/ Cilip 1/ 2002 S.59 f. 
körperlichen Wohlbefindens verbunden ist. ${ }^{26}$ Die Intensität dieser Beeinträchtigung dürfte auch beim freiwilligen Trinken des Brechmittels Ipecacuanha deutlich über die Schmerzen, die mit einer Blutentnahme verbunden sind, hinausgehen.

Gewöhnlich wird die freiwillige Einnahme von Ipecacuanha für weniger gefährlich gehalten. Es gibt jedoch Fälle, in denen sich die Einnahme dieses Mittels verbietet: Als Kontraindikatoren von Ipecacuanha gelten Intoxikationsanzeichen, akute Krankheitszustände, Erkrankungen in den Bereichen des Herz-Kreislaufsystems, Magens, Darms oder der Leber und Nervenerkrankungen sowie Entzündungen, Schleimhauterkrankungen und Tumore im Bereich der Speiseröhre oder des Margen-Darm-Traktes, Herzrhythmusstörungen und hoher Blutdruck. ${ }^{27}$ Bei den beiden letztgenannten Kontraindikationen kann es zu erheblichen Kreislauf- und Herzbeschwerden kommen. Bei entsprechenden Vorschädigungen ist das Eintreten von Rissen in der Magenschleimhaut zu befürchten. ${ }^{28}$

Noch massiver werden die Beeinträchtigungen, wenn der Eingriff gegen den Willen des Betroffenen erfolgt.

Neben den noch näher zu erwähnenden physischen Beeinträchtigungen eines gewaltsamen Brechmitteleinsatzes müssen die psychischen Beeinträchtigungen des Betroffenen, die gleichsam vom Schutzbereich des Art.2 Abs.2 S.1 GG umfasst sind, berücksichtigt werden. ${ }^{29}$ Das gewaltsame Einführen einer Magensonde durch die Nase des Betroffenen kann meist nur erfolgen, wenn dieser von mehreren Personen fixiert wird. Ein solcher Vorgang ist mit erheblichen Beklemmungsgefühlen, ja mit regelrechter Angst verbunden. Die Betroffenen haben das Gefühl staatlicher Gewalt vollkommen ausgeliefert zu sein, was in vielen Fällen schwere Angstzustände auslöst, deren Verarbeitung noch lange Zeit über den konkreten Eingriff andauert. ${ }^{30}$

Darüber hinaus ist der erzwungene Brechmitteleinsatz mit noch erheblicheren gesundheitlichen Gefahren verbunden, wie auch dessen Befürworter einräumen müssen. ${ }^{31}$ Wenn behauptet wird, das Einführen einer Magensonde stelle im medizinischen Bereich inzwischen eine Routinemaßnahme dar, bei der in der Regel keine gesundheitlichen Risiken einträten, ${ }^{32}$ wird unterschlagen, dass das Einführen der Sonde sonst niemals gewaltsam gegen den Willen der Patienten vorgenommen wird. Das gewaltsame Einführen einer Magensonde ist mit erheblichen Gesundheitsgefahren verbunden, wie auch die Hamburger Ärztekammer nach dem Todesfall in Hamburg hervorhob. ${ }^{33}$ Mit Recht weigern sich deshalb viele Ärzte in Krankenhäusern, Beschuldigten in nicht medizinisch indizierten Fällen, brechreizauslösende Mittel zu verabreichen. ${ }^{34}$

26 Benfer, JR 1998, 53 (54).

27 Bachmann/ Püschel/ Sonnen, Kriminalistik 2004, 678 f.

28 Vgl. OLG Frankfurt, NJW 1997, 1647; Birkholz u.a., Kriminalistik 1997, 277 (279).

29 OLG Frankfurt, NJW 1997, 1647 (1648).

30 Vgl. Roggan, Bürgerrechte \& Polizei/ Cilip 1/ 2002 S.60.

31 Bachmann/ Püschel/ Sonnen, Kriminalistik 2004, 678 (679) euphemistisch: »gewisse Bedenken«, vgl. dann aber die bis zur Gefahr des Todeseintritts geschilderten Risiken.

32 Bachmann/ Püschel/ Sonnen, Kriminalistik 2004, 678 (679); Schaefer, NJW 1997, 2437 (2438).

33 Pressemitteilung des Hamburger Ärztekammer v. 30.10.2001.

34 Vgl. Benfer, JR 1998, 53 (54). 
Ruckartige Verteidigungshandlungen gegen das gewaltsame Einführen der Magensonde können beim Beschuldigten zu Verletzungen führen. ${ }^{35}$ Prellungen und Schürfwunden sind durch das gewaltsame Fixieren der Person regelmäßig zu befürchten. ${ }^{36}$

Fälle, in denen die Betroffenen infolge eines Brechmitteleinsatzes die Besinnung verloren, sind bekannt. ${ }^{37}$ In Einzelfällen kann der Einsatz zum Herzstillstand und Tod des Betroffenen führen. ${ }^{38}$ In seltenen Fällen besteht bei stark alkoholisierten oder neurologisch schwer geschädigten Personen sogar die Gefahr eines Reflextodes durch eine übermäßige Reizung von Nervengeflechten des Nervus vagus am Kehlkopfeingang. ${ }^{39}$

Nicht auszuschließen ist auch die Möglichkeit, dass Betroffene Erbrochenes einatmen und u.U. daran ersticken. ${ }^{40}$

Als nicht weniger gefährlich, vielleicht sogar noch gefährlicher, hat sich der Einsatz des Brechmittels Ampomorphin erwiesen. Als Nebenwirkungen des Mittels werden Blutdruckabfall, Atemdepression und krankhafte Schläfrigkeit, sog. Somnolenz, genannt. Bei Überdosierung können Atemlähmung und Krämpfe eintreten. ${ }^{41}$ Auch hier besteht die Gefahr, dass Betroffene an Erbrochenem ersticken.

Als besonders bedenklich werden die Fälle eingeschätzt, in denen beide Mittel kombiniert eingesetzt werden. In diesen Fällen kann es zu nicht übersehbaren Synergieeffekten mit erheblichen Gesundheitsgefährdungen kommen. ${ }^{42}$

Man wird mithin resümieren müssen, dass der Brechmitteleinsatz mit erheblichen Gesundheitsgefahren verbunden ist. ${ }^{43}$

Angesichts dieser gesundheitlicher Risiken muss man bereits feststellen, dass der Brechmitteleinsatz von der Ermächtigungsgrundlage des $\S$ 81a Abs.1 StPO, welche körperliche Eingriffe nur zulässt, wenn keine Nachteile für die Gesundheit der Betroffenen zu befürchten sind, nicht gedeckt ist. Dies ist insbesondere dann der Fall, wenn zuvor - wie im vom OLG Frankfurt zu entscheidenden Fall - eine ärztliche Anamnese hinsichtlich etwaiger gesundheitlicher Risikofaktoren nicht erfolgt ist. ${ }^{44}$

35 Vgl. Amnesty International Jahresbericht 1997 S.177 »Nasenbluten«.

36 Vgl. OLG Frankfurt, NJW 1997, 1647.

37 Vgl. Amnesty International Jahresbericht 1997 S.177.

38 Montgomery, Vorsitzender des Ärzteverbandes Marburger Bund, Interview in: Die Tageszeitung v. 6.1.2005.

39 Bachmann/ Püschel/ Sonnen, Kriminalistik 2004, 678 (679); Roggan, Bürgerrechte \& Polizei/ Cilip 1/2002 S.61.

40 Dallmeyer, StV 1997, 606 (607); Dettmeyer u.a., MedR 2000 S.319; vgl. auch Birkholz u.a., Kriminalistik 1997 S.280, die in Fällen der Aspiration von Erbrochenem auch die Gefahr einer Lungenentzündung feststellten.

41 Dettmeyer u.a., MedR 2000, 316 (319).

42 OLG Frankfurt, NJW 1997, 1647; Dettmeyer u.a., MedR 2000, 316 (320).

43 Binder/ Seemann, NStZ 2002, 234 (236); Dallmeyer, StV 1997, 606 (607); Dettmeyer u.a., MedR 2000, 316 (319); Roggan, Bürgerrechte \& Polizei/Cilip 1/2002 S.65; Wesslau, StV 1997, 341 (342).

44 Vgl. OLG Frankfurt, NJW 1997, 1647 (1649); so auch: Binder/ Seemann NStZ 2002, 234 (235) die einen Brechmitteleinsatz in diesen Fällen zu Recht angesichts der gesundheitlichen Risiken für unzulässig halten. 
Kann eine solche die Gesundheit erheblich gefährdende, nicht der medizinischen Behandlung des Beschuldigten dienende Maßnahme überhaupt »nach den Regeln der ärztlichen Kunst « erfolgen ${ }^{45}$ Die Hamburger Ärztekammer hat hieran zu Recht erhebliche Zweifel und hat sich nach dem Todesfall aus dem Jahr 2001 einstimmig gegen den Brechmitteleinsatz ausgesprochen. ${ }^{46}$

Der Brechmitteleinsatz ist mit der Gefahr erheblicher gesundheitlicher Nachteile verbunden; es dürfte eher ein glücklicher Zufall sein, dass bisher in Deutschland »lediglich« zwei Menschen zu Tode gekommen sind. Auf Grund der gravierenden gesundheitlichen Risiken des Brechmitteleinsatzes ist es auch kaum vorstellbar, dass eine verfassungsgemäße Ermächtigungsgrundlage für diese Maßnahme im Hinblick auf das Recht auf körperliche Unversehrtheit des Art.2 Abs.2 GG geschaffen werden könnte. Die »prozessuale Aufopferungspflicht« des Verdächtigen, welche Ausdruck der Abwägung der Grundrechte der Person und des Strafverfolgungsanspruchs ist, endet dort, wo körperliche Eingriffe mit der Gefahr erheblicher gesundheitlicher Nachteile verbunden sind. ${ }^{47}$

\section{Versto $\beta$ gegen das Verbot des Selbstbelastungszwangs}

Gegen den Brechmitteleinsatz wird des weiteren ein Verstoß gegen das Verbot von Selbstbelastungszwang angeführt. ${ }^{48}$ Dieser Grundsatz »nemo tenetur se ipsum accusare « besagt, dass niemand verpflichtet ist, sich selbst zu belasten und somit an seiner eigenen Überführung mitzuwirken. ${ }^{49}$ Die dogmatische Herleitung des Grundsatzes ist umstritten. Von der Rechtsprechung und Literatur werden eine verfassungsrechtliche Verankerung des Grundsatzes in der Menschenwürde des Art.1 Abs. 1 GG $^{50}$, im allgemeinen Persönlichkeitsrecht des Art. 2 Abs.1 i.V.m. Art. 1 Abs. 1 GG,${ }^{51}$ des Rechts auf informationelle Selbstbestimmung ${ }^{52}$, ebenfalls Art.2 Abs.1 i.V.m. Art.1 Abs.1 GG,

45 Solche Bedenken äußern auch: Binder/ Seemann, NStZ 2002234 (235); Dettmeyer u.a., MedR 2000, 316 (319); Wesslau, StV 1997, 341 (342) sowie hinsichtlich des vom OLG Frankfurt zu entscheidenden Falls: Fahl, JA 1998, 277 (279).

46 Vgl. Pressemitteilung; ferner: Montgomery, Die Tageszeitung 6.1.2005, sowie: Beck, Mitglied im Vorstand des Verbands Demokratischer Ärztinnen und Ärzte, Frankfurter Rundschau v.15.1.2005, der sich für das »nihil nocere«-Prinzip, der Pflicht des Arztes, den Patienten niemals Schaden zuzufügen, als Grenze ärztlichen Tätigwerdens ausspricht.

47 Köhler, ZStW 107 (1995) S.11 (22).

48 So etwa: OLG Frankfurt, NJW 1997, 1647 mit Besprechung von Fahl, JA 1998, 277 u. Wesslau, StV 1997, 341; ferner: Dallmeyer, KritV 2000, 252, 260 ff.; Naucke, StV 2000, 1 (2); Rüping, Das Strafverfahren (1997) S.88; AA etwa: Verrel, NStZ 1997, 415 (419), der gleichwohl gegen die Art und Weise der Beweismittelgewinnung Bedenken äußert und nicht zu Unrecht in Frage stellt, ob für den Eingriff eine hinreichende Ermächtigungsgrundlage besteht.

49 BGHSt 34, 39 (46); OLG Frankfurt, NJW 1996, 1647 (1648); Fahl, JA 1998, 277 (278); vgl. hierzu grundlegend: Neumann, FS für E.A. Wolff (1998) S.373 ff.; Nothhelfer, Die Freiheit von Selbstbezichtigungszwang (1989); Rogall, Der Beschuldigte als Beweismittel gegen sich selbst (1977); H.A. Wolff, Selbstbelastung und Verfahrenstrennung (1997).

50 Naucke, StV 2000, 1; Neumann, FS für E.A. Wolff (1998) S.381.

51 Dallmeyer, KritV 2000, 252 (265).

52 Nothhelfer a.a.O. S.113. 
und im Rechtsstaatsprinzip ${ }^{53}$ vertreten. Der Grundsatz ist elementarer Bestandteil eines fairen Strafverfahrens. ${ }^{54}$ Es spricht einiges dafür, dass man die Herleitung des Grundsatzes nicht auf eine Verfassungsnorm beschränken kann, sondern dass dieser Ausdruck eines Konglomerats unterschiedlicher verfassungsrechtlicher Prinzipien ist. Allgemein anerkannt ist jedenfalls, dass der Grundsatz Verfassungsrang hat.

»Nemo tenetur « ist nicht auf förmliche Vernehmungen beschränkt, sondern schützt den Beschuldigten davor, sich auch auf andere Weise selbst belasten zu müssen. ${ }^{55}$ Der Beschuldigte ist deshalb im Rahmen einer körperlichen Untersuchung zwar zur Duldung der Untersuchung, nicht aber zu einer aktiven Beteiligung an der Untersuchung verpflichtet. $^{56}$

So ist etwa allgemein anerkannt, dass die Bürger nicht verpflichtet sind, bei einer polizeilichen Alkoholkontrolle in das Prüfröhrchen zu blasen. Anschließend darf dem Beschuldigten aber nach ganz überwiegender Auffassung notfalls mit Zwang eine Blutprobe entnommen werden. ${ }^{57}$

Wenn man lediglich unter den »nemo tenetur«-Satz subsumiert, wird man eine Verletzung des Grundsatzes wohl verneinen müssen, da es sich bei dem durch den Brechmitteleinsatz hervorgerufene Sichübergeben nicht um einen willensgesteuerten aktiven Vorgang handelt. ${ }^{58}$

In neuerer Zeit wurden jedoch zunehmend Bedenken gegen die bloße äußere Abgrenzung eines unzulässigen Selbstbelastungszwangs von der notfalls mit Zwang

53 Dallmeyer, KritV 2000, 252 (264); Geppert, FS für Spendel (1992) S.655 (668) Jahn, StV 1998, 653, (654); Paeffgen, Vorüberlegungen zu einer Dogmatik des UntersuchungshaftRechts (1986) S.71.

54 Vgl. Rzepka, Zur Fairness im Deutschen Strafverfahren (2000) S.82 f.; dies., R \& P 2003, 191 (204).

55 Geppert, FS für Spendel (1992) S.655 (668); Neumann, FS für E.A. Wolff (1998) S.377.

56 BGH, NStZ 1996, 502 (504); AK/StPO-Wassermann (1988), §81a Rn.6; Eisenberg, Beweisrecht der StPO (2002) Rn.1627.

57 OLG Düsseldorf, JZ 1988, 984; vgl. hierzu vertiefend: Geppert, FS für Spendel (1992) S.655, 658 ff. Kritisch zur zwangsweisen Blutentnahme etwa: Rossmanith, Die Verfassungsmäßigkeit von körperlichen Eingriffen nach § 81a (1969) S.80.

58 So etwa: Binder/ Seemann, NStZ 2002 S.238; Rogall, NStZ 1998, 66 (67 f.); Wesslau, StV 1997, 341 (342); anderer Auffassung ist das OLG Frankfurt, NJW 1997, 1647 (1648) sowie: Dallmeyer, StV 1997, 606 (608). Etwas anderes könnte sich aus dem Umstand ergeben, dass vor der Verabreichung von Brechmitteln eine ausführliche ärztliche Anamnese für unbedingt erforderlich gehalten wird. In vielen Fällen dürfte eine solche auf Grund von sprachbedingten Verständigungsschwierigkeiten schwer fallen, vgl. den vom OLG Frankfurt, NJW 1997, 1647 geschilderten Fall; ähnliche Bedenken bei: Wesslau, StV 1997, 341 (342). Eine umfassende ärztliche Untersuchung setzt darüber hinaus, regelmäßig die Mitwirkung des Betroffenen an der Untersuchung voraus. Nicht verantwortet werden kann der Brechmitteleinsatz etwa, wenn der Betroffene an den unterschiedlichen bereits benannten Krankheitszuständen leidet. Viele dieser Zustände lassen sich nicht ausschließlich von außen ohne eine aktive Mitwirkung der Betroffenen feststellen. Hier stellt sich das Problem des Zwangs: Kann ein Brechmitteleinsatz gegen den Willen des Beschuldigten vorgenommen werden, wenn dieser sich weigert, die Strafverfolgungsbehörden über eventuell bestehende Risikodispositionen aufzuklären ? 
durchgesetzten zulässigen Informationserhebung durch die Strafverfolgungsbehörden nach dem Kriterium »aktiv - passiv « geäußert. ${ }^{59}$

Ist es konsequent, den Beschuldigten von der Verpflichtung zur aktiven Mitwirkung an einem Alkoholtest freizustellen, ihm aber u.U. im Falle seiner Weigerung gegen seinen Willen unter Gewalteinsatz eine Blutprobe zu entnehmen? ${ }^{60}$

Es dürfte gerade beim Brechmitteleinsatz auch schwer fallen, den Unterschied zwischen aktiver Mitwirkung und passiver Duldung sauber und überzeugend aufzudröseln. ${ }^{61}$ Bereits seit längerem wird kritisiert, dass hinreichend exakte Abgrenzungskriterien bisher nicht zur Verfügung stehen. ${ }^{62}$ Man wird fragen müssen: Kann es im Falle des Brechmitteleinsatzes unter dem Blickwinkel des Verbotes des Selbstbelastungszwangs einen wesentlichen Unterschied machen, ob der Betroffene sich auf Grund einer in ihm aufkommenden massiven Übelkeit zum aktiven Sich-Selbst-Übergeben genötigt sieht oder ob das Mittel einen unüberwindbaren Brechreiz auslöst, dem sich der Verdächtige nicht mehr willensgesteuert, sondern nur noch passiv hingibt $?^{63}$

Eine Bestimmung des Zwecks des Verbots zum Selbstbelastungszwang kann aber weiterhelfen. Zutreffend hat Grünwald das rechtsstaatliche Ziel dieses Grundsatzes herausgearbeitet: »Es darf kein Zwang auf die Willensbildung des Beschuldigten dahin ausgeübt werden, dass er zu seiner Überführung beiträgt. Darum ist (...) auch die Androhung von Übeln gegenüber dem Beschuldigten unzulässig. « ${ }^{64}$

Der Betroffene sieht sich beim Brechmitteleinsatz jedoch mit einer solchen die freie Willensentschließung tangierenden Situation konfrontiert: Er ist auf Grund des »Nemo tenetur«-Grundsatzes zwar nicht verpflichtet, das Brechmittel (aktiv) zu trinken. Wenn er das Brechmittel aber nicht »freiwillig « zu sich nimmt, muss er mit schweren (faktischen) Nachteilen rechnen: In diesem Fall wird ihm - notfalls mit Gewalt - ein Schlauch durch die Nase in den Magen eingeführt und das Brechmittel verabreicht. Wie bereits dargelegt wurde, ist der zwangsweise Brechmitteleinsatz mit weiteren über die freiwillige Brechmittelaufnahme hinausgehenden Gesundheitsrisiken und mit wohl nicht unerheblichen Schmerzen und erheblicher Angst verbunden. Kann wirklich noch davon die Rede sein, dass der Beschuldigte nicht faktisch - im eigenen Interesse zur Minimierung gesundheitlicher Nachteile - zur Mitwirkung an seiner eigenen Belastung gezwungen ist ? »Dem Beschuldigten zu sagen, er sei nicht verpflichtet, das Brechmittel zu schlucken, im Weigerungsfalle werde man es ihm aber gewaltsam verabreichen, grenzt an Zynismus. ${ }^{65}$ Die Ankündigung eines zwangsweise vorgenommen Brechmitteleinsatzes ist geeignet die freie Willensentschließung der Betroffenen erheblich zu beeinträchtigen. ${ }^{66}$ Mit Nachdruck stellt auch Naucke fest: »Im wörtlichen

59 Vgl. etwa: Verrel, NStZ 1997, 415 (418); Wesslau, StV 1997, 341 (342 f.) und grundlegend: Neumann, FS für E.A. Wolff (1998) S.373 ff.

60 So schon Neumann, FS für E.A. Wolff (1998) S.374.

61 Neumann, FS für E.A. Wolff (1998) S.375.

62 Fahl, JuS 2001, 47 (52); Hacketal, JR 2001, 164 (165).

63 So insbesondere auch: Wesslau, StV 1997, 341 (343) und auch Neumann a.a.O. S.375.

64 Grünwald, JZ 1981, 423 (428).

65 Neumann, FS für E.A. Wolff (1998) S.375 f.

66 Neumann, FS für E.A. Wolff (1998) S.381. 
Sinne zwanghaft herausgepresste Beweismittel sind der Kernfall des Verstoßes gegen die Selbstbelastungsfreiheit. $\ll^{67}$

\section{Verstoß gegen die Menschenwürde}

Gegen den Brechmitteleinsatz wird darüber hinaus mit gewichtiger Begründung eine Verletzung der Menschenwürde des Art.1 Abs.1 GG geltend gemacht. Unter der Menschenwürde versteht das BVerfG den sozialen Wert- und Achtungsanspruch, der dem Mensch wegen seines Menschseins zukommt. ${ }^{68}$ Zur Bestimmung einer Verletzung der Menschenwürde wird die von Dürig in Anschluss an Kant ${ }^{69}$ entwickelte Objektformel herangezogen: Ein Verstoß gegen die Menschenwürde liegt danach vor, wenn der Mensch zum bloßen Objekt staatlichen Handelns gemacht wird. Es verbietet sich, den Menschen einer Behandlung auszusetzen, die seinen Subjektstatus prinzipiell in Frage stellt. ${ }^{70}$ Es sollte deshalb eine Selbstverständlichkeit sein, dass der Beschuldigte nicht zum bloßen Objekt der Strafverfolgung degradiert werden darf. ${ }^{71}$ Eine Wahrheitsfindung um jeden Preis gibt es im Rechtsstaat nicht. ${ }^{72}$ Einer Person mit Gewalt eine Magensonde einzuführen, welche ihr Brechmittel und große Wassermengen gegen ihren Willen zuführt, um diese zum Erbrechen zu zwingen, bedeutet den Willen dieser Person, das Recht über den eigenen Körper zu verfügen, zu missachten. Von der Autonomie des Betroffenen, über die eigenen Körperfunktionen zu bestimmen, kann dann nicht mehr die Rede sein. ${ }^{73}$ Der Körper des Betroffenen wird zum »Gegenstandslieferanten ${ }^{\text {" herabgesetzt. }}{ }^{74}$ Wenn der Hessische Generalstaatsanwalt Schaefer behauptet, hierbei gehe es nur um eine Frage der » ̈̈sthetik $«,{ }^{75}$ drängt sich der Eindruck auf, dass dieser einer Verbrechensbekämpfungslogik verhaftet ist, welche die Grundrechte der Betroffenen gegenüber dem erklärten Ziel einer effektiven Strafrechtspflege ausgesprochen gering schätzt.

Tatsächlich stellt das zwangsweise Erbrechen nämlich eine erhebliche Demütigung der Betroffenen dar, welche geeignet ist, die Subjektqualität der Person in Frage zu stellen. Der Verdächtige droht zum bloßen Verfahrensgegenstand herabgestuft zu werden.

67 Naucke, StV 2000, 1 (2).

68 BVerfGE 87, 209 (228).

69 Kant, Metaphysik der Sitten (Weischedel 1977) S.453 »(..) denn der Mensch kann nie bloß als Mittel zu den Absichten eines anderen gehandhabt und unter die Gegenstände des Sachenrechts gemengt werden, wowider ihn seine angeborne Persönlichkeit schützt (...)«

70 Dürig, AöR 81 (1956), 117 (127); BVerfGE 50, 166 (175); BVerfGE 87, 209 (228); sowie OLG Frankfurt, NJW 1997, 1647 (1648) unter Bezugnahme auf den Kantischen Ausspruch »Der Mensch muss immer Zweck an sich selbst bleiben.«

Diese Definition wird nicht selten als »wenig scharfkantig « kritisiert, überzeugendere Kriterien, die einen Menschenwürdeverstoß präziser fassen könnten, stehen freilich nicht zur Verfügung; Bock, Begriff, Inhalt und Zulässigkeit der Beweislastumkehr im materiellen Strafrecht (2001) S.233; Neumann, FS für E.A. Wolff (1998) S.384.

71 Vgl. nur: Benfer, JR 1998, 53 (54); Fahl, JuS 2001, 47 (52); Meyer-Goßner, StPO (2003), Einl. Rn.80.

72 BGHSt 14, 358 (365).

73 So auch: Amelung/ Wirth, StV 2002, 161 (167).

74 Zaczyk, NStZ 2002 S.126.

75 Schaefer, NJW 1997, 2437 (2438). 
Der Brechtmitteleinsatz verletzt die Betroffenen mithin in ihrer Menschenwürde aus Art. 1 Abs. 1 GG. ${ }^{76}$

\section{Verstoß gegen das Misshandlungsverbot des Art.104 Abs.1 S.2 GG}

Neben einer Verletzung des Art.1 Abs.1 GG könnte der Brechmitteleinsatz darüber hinaus eine Verletzung von Art.104 Abs.1 S.2 GG darstellen. Danach dürfen festgehaltene Personen weder seelisch noch körperlich misshandelt werden. Bei dieser Vorschrift handelt es sich um eine Konkretisierung von Art.1 Abs.1 GG. Wie Art.1 Abs.1 GG ist auch der Art.104 Abs.1 S.2 GG vorbehaltlos garantiert. ${ }^{77}$ Das Misshandlungsverbot trägt der hohen faktischen Abhängigkeit von Personen, die von staatlicher Seite festgehalten werden, Rechnung. ${ }^{78}$ Als zentrale Ausprägungen des Art.104 Abs.1 S.2 GG werden der Schutz vor Folter und demütigenden Behandlungen genannt. ${ }^{79}$

Cornelia Sonntag-Wolgast, Vorsitzende des Innenausschusses im Bundestag, und eine Reihe anderer Stimmen machen geltend, beim Brechmitteleinsatz handele es sich um eine Maßnahme, die mit dem Einsatz von Folter vergleichbar sei. ${ }^{80}$ Bei der Äußerung von Foltervorwürfen ist jedoch Vorsicht geboten: Folter zeichnet sich dadurch aus, dass einer Person final Leiden zugefügt werden, um auf diese Weise Aussagen zu erpressen. ${ }^{81}$ Von einer finalen Leidzufügung und somit von Folter kann im Fall des Brechmitteleinsatzes nicht die Rede sein. Der zwangsweise Brechmitteleinsatz ist aber faktisch untrennbar mit schwerwiegenden Demütigungen und der Verursachung von erheblichen Schmerzen für die festgehaltene Person verbunden. Solche physischen und seelischen Misshandlungen werden ebenfalls von Art.104 Abs.1 S.2 GG untersagt. ${ }^{82}$

76 OLG Frankfurt, NJW 1997, 1647 (1648); Amelung/ Wirth, StV 2002, 161 (167); Dallmeyer, StV 1997, 606 (610); ders., KritV 2000, 252 (259); Kühne, Strafprozessrecht (2003) S.247; Naucke in: Landwehr (Hrsg.), Freiheit, Gleichheit, Selbständigkeit (1999) S.89 (100 f.); Roxin, Strafverfahrensrecht (1998) S.274; Schorn, Schutz der Menschenwürde im Strafverfahren (1963) S.110 f. sowie nicht ausdrücklich auf den Brechmitteleinsatz bezogen: Neumann, FS für E.A. Wolff (1998) S.392, soweit körperliche Eingriffe über bloße Routinemaßnahmen hinausgehen. Vgl. hierzu auch bereits die erheblichen Benken von: Beling, Die Beweisverbote als Grenzen der Wahrheitsfindung im Strafprozess (1903) S.11 f.

77 Dreier-GG-Schulze-Fielitz (2000), Art. 104 Rn.54.

78 v. Mangold/Klein/Stark - Gusy (2001) Rn.31.

79 Dreier-GG-Schulze-Fielitz (2000), Art.104 Rn.54.

80 Vgl. Die Tageszeitung v. 11.1.2005, http://www.taz.de/pt/2005/01/11/a0068.nf/textdruck; Dallmeyer, KritV 2000, 252 (259); Roggan, Bürgerrecht \& Polizei/ Cilip 1/2002 S.59 (65); vgl. auch schon: Sax a.a.O. S.984 f. »Art moderner Folter« hinsichtlich des vergleichbaren zwangsweise vorgenommenen Magenaushebens.

81 Dreier-GG-Schulze-Fielitz (2000) Art.104 Rn.55.

82 Vgl. Maunz/Dürig/Herzog-Dürig, Art.104 Rn.20. Z.T. wird gefordert, dass das Misshandlungsverbot nur vor finalen Misshandlungen schütze, vgl. Pieroth/ Jarass, GG (2004), Art.104 Rn.8. Eine solche Vorausstzung dürfte über die Fälle der Folter hinaus, jedoch praktisch nie vorliegen. Art.104 Abs.1 S.2 GG muss deshalb weiter ausgelegt werden und auch vor solchen Behandlungen schützen, die praktisch immer faktisch mit erheblichen körperlichen und seelischen, über die bloße Freiheitsentziehung hinausgehenden (vgl. v.Mangold/Klein/Starck-Gusy (2001), Art.104 Rn.32) Beeinträchtigungen verbunden sind. 
Die Menschenrechtsorganisation Amnesty International (AI) hatte sich deshalb bereits 1997 vehement gegen den Brechmitteleinsatz ausgesprochen: »Nach Auffassung von Amnesty International stellt die zwangsweise Verabreichung von Brechmitteln, sofern sie nicht aus medizinischen Gründen erforderlich ist, eine grausame, unmenschliche oder erniedrigende Behandlung dar. $«{ }^{83}$ Diese Vorwürfe werden von AI im Zusammenhang mit dem Bremer Todesfall zu Recht erneut erhoben. ${ }^{84}$ Der gewaltsam vorgenommene Brechmitteleinsatz dürfte im Einzelfall regelmäßig die Schwelle der durch Art.104 Abs.1 S.2 GG absolut verbotenen Misshandlungen überschreiten.

\section{Verhältnismäßigkeitsprinzip}

Fragwürdig ist außerdem, auch wenn man den obigen Ausführungen nicht in allen Punkten folgt, ob der Brechmitteleinsatz noch als verhältnismäßig angesehen werden kann. Die Verhältnismäßigkeit einer Maßnahme setzt voraus, dass das mit der geeigneten Maßnahme verfolgte legitime Ziel nicht mit milderen Mittel verfolgt werden kann und dass der Einsatz angemessen ist.

Der Brechmitteleinsatz könnte schon deshalb unverhältnismäßig sein, weil mildere Mittel zur Verfügung stehen. Es wäre insbesondere denkbar, den Verdächtigten so lange in Haft zu halten, bis der Mageninhalt auf natürlichem Wege ausgeschieden wird ${ }^{85}$ Dies ist zwar auch mit erheblichen Eingriffen in die Freiheit der Person verbunden, die nur bei einem hohen Verdachtsgrad zu rechtfertigen sind; hierbei handelt es sich aber angesichts der verfassungsrechtlichen Bedenken gegen den Brechmitteleinsatz um ein milderes Mittel.

Dass es sich bei solchen Überlegungen nicht um bloße »graue Theorie« handelt, zeigt das Bundesland Bayern, in dem im Hinblick auf das Verhältnismäßigkeitsprinzip auf Brechmitteleinsätze verzichtet wird. ${ }^{86}$

Darüber hinaus bestehen hinsichtlich der Angemessenheit des Brechmitteleinsatzes erhebliche Bedenken. Die Brechmittelfälle deuten darauf hin, dass die Strafverfolgungsorgane in vielen Fällen im »Drogenkrieg « das erforderliche Maß aus den Augen verloren haben. Einen bloß Tatverdächtigen wegen geringer vermuteter Rauschgiftmengen einer derart gefährlichen und schmerzhaften Behandlung zu unterziehen, kann nicht mehr als angemessen angesehen werden. ${ }^{87}$

\section{Alternativen zu einer Drogenpolitik der Härte}

Die weitgehend erfolglosen Versuche, den Drogenmissbrauch in Deutschland, aber auch in anderen westlichen Industrienationen, mittels einer Politik der Härte massiv

83 Amnesty International Jahresbericht 1997, $177 \mathrm{f}$.

84 Vgl. Frankfurter Rundschau v. 8.1.2005.

85 OLG Frankfurt, NJW 1997, 1647 (1649); Binder/ Seemann, NStZ 2002, 236 f.; Dettmeyer u.a., MedR 2000, 316 (318); Montgomery, Die Tageszeitung v. 6.1.2005; Roggan, Bürgerrechte \& Polizei/ Cilip 1/2002 S.63.

86 Frankfurter Rundschau v. 8.1.2005.

87 Binder/ Seemann, NStZ 2002 S.237; Wesslau, StV 1997, 341 (342) sowie Lesch, Strafprozessrecht (1999) S.182 u. Meyer-Goßner, StPO (2003) Rn.22 bei »Kleindealern«. 
einzudämmen und das durch eine Kriminalisierung mitverursachte Elend vieler Drogenabhängiger fordern dazu heraus, neue Wege im Bereich der Drogenpolitik zu beschreiten. ${ }^{88}$

Wird Strafrecht als Verbrechens- bzw. Verbrecherbekämpfungsrecht verstanden, drohen darüber hinaus rechtsstaatliche Fundamentalprinzipien nicht selten aus dem Blickwinkel zu geraten. ${ }^{89}$ Dies zeigt sich paradigmatisch im heutigen Betäubungsmittelstrafrecht. Hier ist von einem internationalen »War on Drugs « die Rede. Im »Krieg« gegen den »Drogensumpf « werden Tatverdächtige nicht selten vorschnell als »Drogendealer ${ }^{90}$ und $»$ Schwerstverbrecher $«$ die auch körperliche Nachteile hinnehmen müssten, ${ }^{91}$ vorverurteilt. Die archaischen Zeiten, in denen das Strafrecht als Mittel zur physischen Vernichtung des inneren »Feindes« verstanden wird, sollten überwunden sein. Nicht selten stellen sich Verdächtigungen zusätzlich nachträglich als falsch heraus. So förderte eine neue Studie über die Praxis des Brechmitteleinsatzes in Hamburg zu Tage, dass in lediglich 63,9 \% der Fälle sich der Tatverdacht als richtig herausstellte. $^{92}$

Es wird Zeit, dass sich die Verantwortlichen im Bereich der Drogenpolitik auf die Grundprinzipien einer rechtsstaatlichen Strafrechtspflege besinnen. Dazu gehört, dass es eine »Verbrechensbekämpfung um jeden Preis« im Rechtsstaat nicht geben darf; insbesondere Menschenwürde und fundamentale rechtsstaatliche Verfahrensprinzipien dürfen als Unverfügbares im Strafrecht ${ }^{93}$ im Interesse der Betroffenen, aber auch im Interesse gesellschaftlicher Zivilität nicht angetastet werden. Der zwangsweise Brechmitteleinsatz ist mit diesen Prinzipien nicht zu vereinbaren und sollte deshalb als strafprozessuale Ermittlungsmethode abgeschafft werden.

88 Vgl. hierzu die Überlegungen von: Baratta, KrimJ 1990 S.2 ff.; Böllinger in: WE Kriminalpolitikforschung (Hrsg.), Legalisierung von Heroin - Die neue Debatte, Internationales Symposium an der Universität Bremen (1992) S.47 ff.; Hassemer, KritV 1993, 198 ff.; Kerner in: Kaiser u.a. (Hrsg.), Kleines Kriminologisches Wörterbuch (1993) S.93 ff., bes. S.99; Neskovic in: Gössner (Hrsg.), Mythos Sicherheit (1995) S.351 ff. sowie mit zahlreichen weiteren Nachweisen auf das heutige Schrifttum: P.A. Albrecht, Kriminologie (2002) S.342 ff., 353 ff. und schließlich die Beiträge in Forum Recht 2/2003 zum Schwerpunkt Drogenpolitik.

89 Gegen eine solche Fehlinterpretation von Strafrecht und Polizeirecht als Kampfinstrumente: Harzer, Jura 1995, 208 (210 Fn.7); Kreuzer, FS für Blau (1985) S.459 (462); Lisken, ZRP 1994, 49 ff.; Marxen, KritV 1990, 287 (290); Naucke, KritV 1990, 244 (247).

90 Benfer, JR 1998, 53.

91 So der Bremer Innensenator s.o.

92 Bachmann/ Püschel/ Sonnen, Kriminalistik 2004, 678 (682).

93 Vgl. die Überlegungen von Hassemer, FS für Maihofer (1988) S.183 ff. 


\section{Neu bei Nomos}

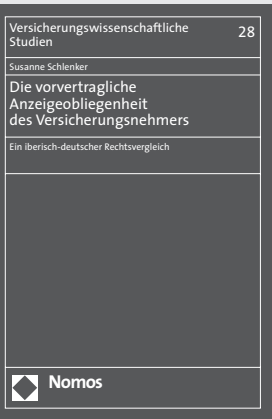

\section{Die vorvertragliche Anzeigeobliegenheit des Versicherungsnehmers}

Ein iberisch-deutscher Rechtsvergleich

Von RAin Dr. Susanne Schlenker

2005, 177 S., brosch., 34,- €, ISBN 3-8329-1074-3

(Versicherungswissenschaftliche Studien, Bd. 28)

In Deutschland soll das Versicherungsvertragsrecht geändert werden und damit auch die vorvertragliche Anzeigeobliegenheit. Die Darstellung der Rechtslage in Spanien mit seinem modernen Versicherungsvertragsgesetz und in Portugal, wo die Normen des 19. Jahrhunderts gelten, soll als Arbeitsgrundlage für mögliche Änderungen dienen.

In Kapiteln werden rechtsvergleichend die technischen Grundlagen bzw. die Bedeutung der vorvertraglichen Risikobestimmung herausgearbeitet, die historische Entwicklung in den drei Ländern dargestellt, die relevanten Vorschriften und die Rechtsprechung ausführlich erläutert, das Verhältnis der Regelungen zum allgemeinen Zivilrecht diskutiert sowie die Besonderheiten in der Haftpflicht- und Lebensversicherung aufgezeigt.

Wer sich für die aktuelle Gesetzesänderung oder für die spanische und portugiesische Rechtsordnung speziell in Bezug auf das Versicherungsvertragsrecht, aber auch im Hinblick auf das allgemeine Zivilrecht interessiert, findet in dieser Arbeit umfassende Informationen. 